\title{
Non-ribosomal peptide synthetase module fusions to produce derivatives of daptomycin in Streptomyces roseosporus
}

\begin{abstract}
Correspondence
Richard H. Baltz

rbaltz@cubist.com
\end{abstract}

Received 19 May 2008

Revised 19 June 2008

Accepted 23 June 2008

\author{
Sascha Doekel, Marie-Françoise Coëffet-Le Gal, Jian-Qiao Gu, Min Chu, \\ Richard H. Baltz and Paul Brian
}

Cubist Pharmaceuticals Inc., 65 Hayden Avenue, Lexington, MA 02421, USA

\section{INTRODUCTION}

Streptomyces roseosporus NRRL 15998 produces the A21978C complex of acidic lipopeptides of the daptomycin family, which consist of at least six factors with different fatty acids attached to the amino group of $\mathrm{L}-\operatorname{Trp}_{1}$ of the peptide (Baltz et al., 2005; Debono et al., 1987). (The subscripts refer to positions of amino acids in the peptide, starting with $\mathrm{L}-\mathrm{Trp}_{1}$, as discussed below and in Fig. 1b.) The three major fermentation components of the A21978C complex have 11-, 12- and 13-carbon branched-chain fatty acids, whereas the minor factor daptomycin, which can be enriched by feeding decanoic acid during fermentation (Baltz et al., 2005; Huber et al., 1988), has a 10-carbon straight chain (Fig. 1a). Daptomycin has been approved as Cubicin ${ }^{\circledR}$ (daptomycin-for-injection) for treatment of skin and skin structure infections caused by Gram-positive pathogens (Arbeit et al., 2004), and for bacteraemia and endocarditis caused by Staphylococcus aureus, including strains resistant to meticillin (MRSA) (Fowler et al., 2006).

Abbreviations: A, adenylation; C, condensation; CDA, calcium-dependent antibiotic; E, epimerization; ESI, electrospray ionization; D-hAsn, Dhydroxyasparagine; D-hPhg, D-hydroxyphenylglycine; HR-MS, high resolution mass spectrometry; Kyn, kynurenine; LC-MS, liquid chromatography-mass spectrometry; 3mGlu, 3-methylglutamic acid; mOAsp, methoxyaspartic acid; NRPS, non-ribosomal peptide synthetase; PCP, peptidyl carrier protein; Sar, sarcosine; T, thiolation; Te, thioesterase.

A supplementary table of primers is available with the online version of this paper.
The daptomycin biosynthetic gene cluster was cloned and sequenced, and the non-ribosomal peptide synthetase (NRPS) and other genes identified (Miao et al., 2005; Fig. 1b). The cyclic, branched, 13-membered lipopeptide backbone of daptomycin is produced by three giant NRPS multi-enzymes, DptA, DptBC and DptD, encoded by the $d p t A, d p t B C$ and $d p t D$ genes, respectively. NRPSs are composed of conserved repeating units (modules) each with an average size of $120 \mathrm{kDa}$ (Sieber \& Marahiel, 2005). Each module catalyses the incorporation of one specific amino acid in a sequence that is collinear with the modular organization in the DNA coding sequences. A typical module has three catalytic domains, responsible for peptide bond formation (condensation domain, C; Stachelhaus et al., 1998), recognition and activation of substrate amino acids (adenylation domain, A; Stachelhaus et al., 1999), and translocation (thiolation domain, T; or peptidyl carrier protein, PCP; Stachelhaus et al., 1996). Modules may also contain accessory domains, such as epimerization (E) domains that catalyse the conversion of L-amino acids to D-isomers (Stachelhaus \& Walsh, 2000). The terminal modules of NRPSs often have thioesterase (Te) domains involved in cleavage of the nascent peptide, releasing linear or cyclic peptides, or cyclic depsipeptides (Kohli \& Walsh, 2003). Such modules are arranged as C-A-T-Te, and individual enzyme domains are connected by linker peptides.

Because of their collinear gene/protein module organization, NRPSs present promising targets for combinatorial 
(a)
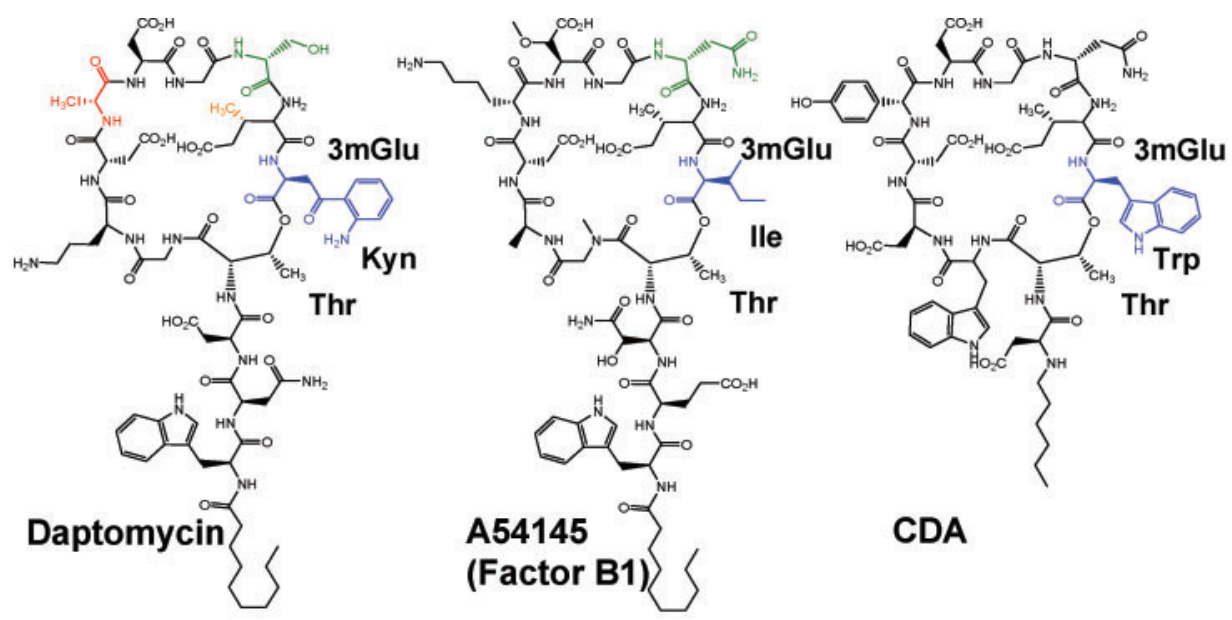

CDA

(b)

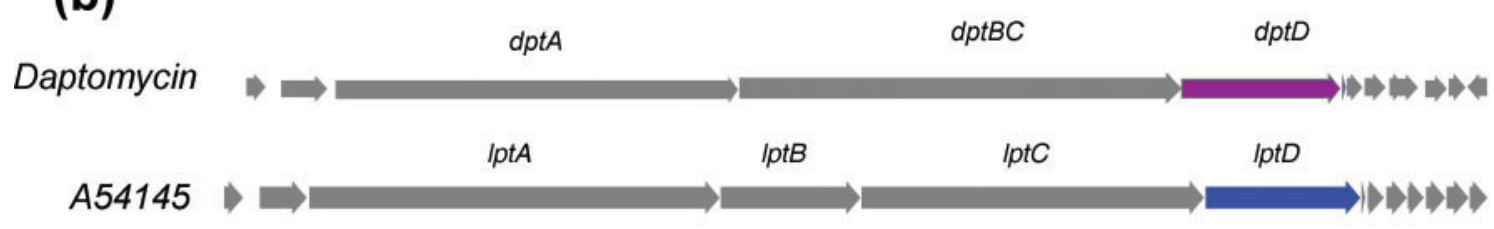

$C D A$

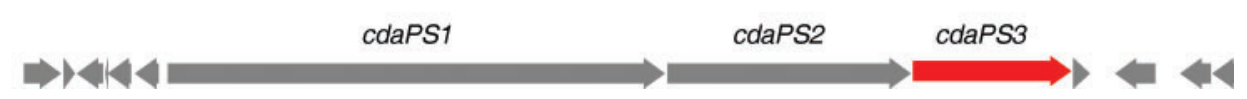

Fig. 1. Structures of lipopeptide antibiotics and organization of NRPS genes. (a) Chemical structures of daptomycin, A54145 (factor B1) and CDA. (b) Organization of the NRPS gene clusters of daptomycin, A54145 and CDA. The $\operatorname{dptA}, d p t B C$ and $d p t D$ genes encode multi-modular NRPS enzymes, DptA, DptBC and DptD, that sequencially incorporate L-Trp ${ }_{1}-\mathrm{D}-\mathrm{Asn}_{2}-\mathrm{L}-$ $\mathrm{Asp}_{3}-\mathrm{L}-\mathrm{Thr}_{4}-\mathrm{Gly}_{5}, \mathrm{~L}-\mathrm{Orn}_{6}-\mathrm{L}-\mathrm{Asp}_{7}-\mathrm{D}-\mathrm{Ala}_{8}-\mathrm{L}-\mathrm{Asp}_{9}-\mathrm{Gly}_{10}-\mathrm{D}-\mathrm{Ser}_{11}$ and L-3mGlu ${ }_{12}-\mathrm{L}-\mathrm{Kyn}_{13}$, respectively. The IptA, IptB, IptC and IptD genes encode LptA, LptB, LptC and DptD that incorporate L-Trp ${ }_{1}-\mathrm{D}-\mathrm{Glu}_{2}-\mathrm{L}-\mathrm{Asn}_{3}-\mathrm{L}-\mathrm{Thr}_{4}-\mathrm{Sar}_{5}, \mathrm{~L}-\mathrm{Ala}_{6} \mathrm{~L}-\mathrm{Asp} \mathrm{s}_{7}, \mathrm{D}-\mathrm{Lys} \mathrm{s}_{8}-\mathrm{L}-$ $\mathrm{mOAsp}_{9}-\mathrm{Gly}_{10}-\mathrm{D}-\mathrm{Asn}_{11}$ and L-3mGlu ${ }_{12}-\mathrm{L}-\mathrm{Il}_{13}$, respectively. The cdaPS1, cdaPS2 and cdaPS3 genes encode CdaPS1, CdaPS2 and CdaPS3 that incorporate L-Ser ${ }_{1}-\mathrm{L}-\mathrm{Thr}_{2}-\mathrm{D}-\mathrm{Trp}_{3}-\mathrm{L}-\mathrm{Asp}_{4}-\mathrm{L}-\mathrm{Asp}_{5}$-D-hPhg ${ }_{6}, \mathrm{~L}-\mathrm{Asp}_{7}-\mathrm{Gly}_{8}$-D-hAsn and L-3mGlu $_{10}$-L$\operatorname{Trp}_{11}$, respectively. (See Baltz, 2008; Baltz et al., 2005.)

biosynthesis (Baltz, 2008; Baltz et al., 2005; Fischbach \& Walsh, 2006; Sieber \& Marahiel, 2005). Module exchanges in NRPS model systems have proven to be useful to generate peptides of a targeted sequence (Doekel \& Marahiel, 2000; Mootz et al., 2000), and several novel derivatives of surfactin have been produced by the targeted replacement of modules (Mootz et al., 2002; Stachelhaus et al., 1995; Yakimov et al., 2000). In other in vivo studies, the specificity-conferring codes in the active sites of A domains (Stachelhaus et al., 1999; Challis et al., 2000) have been exploited to redirect surfactin biosynthesis by site-specific mutagenesis (Eppelmann et al., 2002). Biochemical investigations on NRPSs have helped define rules of substrate specificity and promiscuity, and to define inter-domain linkers and inter-peptide docking domains, thereby providing tools to guide the engineering of NRPS biosynthetic genes to produce novel peptides in vivo (Fischbach \& Walsh, 2006; Sieber \& Marahiel, 2005).

The enzymic domains of NRPSs are connected by peptide linkers that have no apparent sequence conservation. The linkers have been used in vitro (Doekel \& Marahiel, 2000; Mootz et al., 2000) and in vivo (Stachelhaus et al., 1995; Nguyen et al., 2006b) to engineer modular NRPSs by module and domain exchanges. Production levels of novel lipopeptides related to daptomycin, engineered by single module exchanges, ranged from 17 to $50 \%$ of controls (Nguyen et al., 2006b).

The functions of NRPS inter-domain linkers have not been extensively explored, although there has been speculation about a role in initiation of protein-protein interactions (Shen et al., 2002). The 18 aa linker connecting the T (or PCP) and downstream $\mathrm{C}$ domains in a T-C di-domain of the tyrocidine synthetase TycC apparently exhibits considerable conformational flexibility, and the final seven amino acids lack defined interactions with either domain and are mobile in the PCP-C crystal structure (Samel et al., 2007). The flexibility in this linker reflects the requirement for the $\mathrm{T}$ (PCP) to interact with the A domain and the preceding and following $\mathrm{C}$ domains to function in peptide assembly. 
Daptomycin production can be restored in a $d p t D$ deletion mutant of $S$. roseosporus $(\Delta d p t D)$ with $d p t D$ expressed under the transcriptional control of the ermEp* promoter by trans-complementation at an ectopic site in the chromosome (Coëffet-Le Gal et al., 2006; Miao et al., 2006b). This $\Delta d p t D$ mutation can also be complemented by the genes $l p t D$ and $c d a P S 3$, encoding the terminal subunits from the distantly related lipopeptide biosynthetic pathways of A54145 and calcium-dependent antibiotic (CDA), respectively, to produce novel hybrid lipopeptides (Baltz, 2008; Coëffet-Le Gal et al., 2006; Miao et al., 2006b; Nguyen et al., 2006b). The analogues contained Trp or Ile/ Val in place of $\mathrm{Kyn}_{13}$ when subunits CdaPS3 (Hojati et al., 2002) or LptD (Miao et al., 2006a) were exchanged for the native DptD (Fig. 1a). This work indicated that the Te domains from the CdaPS3 and LptD subunits were able to cyclize heterologous peptides differing substantially from the native CDA and A54145 peptides, consistent with in vitro chemoenzymic studies with excised $\mathrm{T}$-Te di-domains that generated lipopeptides related to daptomycin (Grünewald et al., 2004; Kopp et al., 2006). In the in vivo studies, the recombinants produced daptomycin derivatives containing $\operatorname{Trp}_{13}$ or $\mathrm{Ile}_{13} / \mathrm{Val}_{13}$ in yields of about $50 \%$ and $25 \%$ of control, respectively (Coëffet-Le Gal et al., 2006; Miao et al., 2006b). The reduction in yields might be due to suboptimal interpeptide docking, substrate recognition or ring closure.

This heterologous whole-subunit trans-complementation system for combinatorial biosynthesis of daptomycin analogues provides a means to test parameters important for high productivity of engineered NRPSs. In particular, it can be used to explore the efficiency of producing hybrid molecules by fusing modules or exchanging domains from di-modular DptD, LptD and CdaPS3 subunits to engineer positions 12 and 13 . This also has a practical advantage in that positions 12 and 13 are important determinants for antibacterial activity (Coëffet-Le Gal et al., 2006; Miao et al., 2006b; Nguyen et al., 2006a).

In the present study, we generated module fusions at T-C linkers, and showed that the linker region can be modified by amino acid substitutions, and by deletion or insertion of four amino acids, without disrupting lipopeptide production. We also generated a functional domain exchange by fusing a heterologous $\mathrm{C}-\mathrm{A}$ di-domain to the $\mathrm{T}-\mathrm{Te}$ didomain of DptD at the A-T linker, and the recombinant produced a novel derivative of daptomycin with $\mathrm{Asn}_{13}$.

\section{METHODS}

Strains, growth conditions and conjugal transfer of plasmids. Strains and plasmids are listed in Table 1. S. roseosporus was grown on trypticase soy (TS) agar or in TS broth at $30{ }^{\circ} \mathrm{C}$. AS-1 agar and fermentation medium F10A were described previously (Miao et al., 2006b). Plasmids were transferred from Escherichia coli ML22 into S. roseosporus UA378 $(\Delta d p t D)$ by conjugation (Miao et al., 2006b). Exconjugants were selected for hygromycin resistance at $50 \mu \mathrm{g} \mathrm{ml}^{-1}$ on AS-1 agar.
Antibiotic production, purification and assay. S. roseosporus strains containing recombinant plasmids were fermented in triplicate for 5 days as described previously (Miao et al., 2006b) and supernatants of fermentation broths were tested for antibacterial activity. Samples $(100 \mu \mathrm{l})$ were added to wells $(5 \mathrm{~mm}$ diameter) embedded in AS-1 agar plates supplemented with $10 \mathrm{mM} \mathrm{CaCl}_{2}$ and Staphylococcus aureus $42\left(10^{5}\right.$ c.f.u. $\left.\mathrm{ml}^{-1}\right)$. After incubation at $37{ }^{\circ} \mathrm{C}$ overnight, the plates were inspected for zones of inhibition, and MICs were determined (Miao et al., 2006b). Lipopeptides were quantified from broth cultures by HPLC and LC-MS using daptomycin as the standard, and were purified as described by Nguyen et al. (2006a).

Plasmid constructions. Expression plasmids for engineered $d p t D$ genes were constructed based on pRB04, a pHM11a-derived plasmid in which the $d p t D$ gene is expressed from the strong, constitutive ermEp $p^{*}$ promoter (Miao et al., 2006b). The coding sequence of $d p t D$ was cloned by inserting a $B s r \mathrm{GI} / \mathrm{BamHI}$ (blunted) fragment (primers CB312 and CB313). Primer CB313 (see Supplementary Table S1, available with the online version of this paper) contained artificial AvrII and PmeI sites that were positioned in the A-site of the T-C linker of $d p t D$ as shown in Fig. 2(b), giving rise to plasmid pCB301. This cloning step was repeated using primer pairs CB312/CB314 and CB312/CB315, respectively, to shift the AvrII site downstream to the B and $\mathrm{C}$ sites of the T-C linker (plasmids pCB302 and pCB303, respectively). Plasmid pCB302 was used to ligate in-frame an AvrII/ $H p a$ I fragment comprising the terminal modules (CATTe) for $d p t D 2$ (Kyn), lptD2 (Ile) and cdaPS3-2 (Trp) (primer pairs CB323/CB325, CB327/CB329 and CB330/CB331) generating plasmids pCB304, pCB305 and pCB306, respectively. Plasmids pCB301 and pCB303 were used for cloning a $d p t D 2$ fragment with varying 5 ' terminal ends to accommodate $\mathrm{A}$ and $\mathrm{C}$ sites of the $\mathrm{T}-\mathrm{C}$ linker, generating plasmids pCB307 and pCB308 (using primer pairs CB322/CB325 for the A site and CB324/CB325 for the C-site, respectively). Plasmids pCB309 and pCB310 were constructed as above, but by switching the inserts to generate a deletion or insertion of $12 \mathrm{nt}$ in the linker coding sequences (Table 1). C-A di-domains for $d p t D 2$ and $l p t C 4$ were amplified using primer pairs CB323/CB354 and CB355/CB356, respectively, and cloned into pCB302. CAT modules and C-A didomains were generated using $\lambda$ Red-mediated recombination (Datsenko \& Wanner, 2000; Nguyen et al., 2006b; Zhang et al., 1998) in E. coli DH10B(pKD78) using the following primers: CB346/ CB347 for $d p t D 2(\mathrm{CAT}), \mathrm{CB} 346 / \mathrm{CB} 357$ for $d p t \mathrm{D} 2(\mathrm{CA}), \mathrm{CB} 352 / \mathrm{CB} 353$ for $l p t C 4(\mathrm{CAT})$ and $\mathrm{CB} 352 / \mathrm{CB} 358$ for $l p t C 4(\mathrm{CA})$. The Te domain from $d p t D$ was amplified using the primers CB332 and CB333 and subcloned along with spec (amplified with CB357/CB358) using PmeI and HindIII to form the Te/spec cassette. This cassette was excised and cloned into HpaI/HindIII sites in conjunction with the CAT modules of Kyn and Asn, respectively, to give pCB401 (Kyn) and pCB407 (Asn). The T-Te di-domain from $d p t D$ was amplified using the primers CB359 and CB333 and subcloned along with spec (amplified with CB357/CB358) using PmeI and HindIII to form the TTe/spec cassette. This cassette was excised and cloned into HpaI/HindIII sites in conjunction with the C-A di-domains of Kyn and Asn, respectively, to give pCB409 (Kyn) and pCB410 (Asn). Cloning junctions were sequenced for confirmation.

\section{RESULTS}

\section{Module fusions and domain exchanges in NRPS subunit DptD}

Previous successful whole-subunit trans-complementation studies, using the di-modular $\mathrm{DptD}$, and distantly related LptC and CdaPS3 (Baltz, 2008; Coëffet-Le Gal et al., 2006; 
Table 1. Strains and plasmids

\begin{tabular}{|c|c|c|}
\hline \multicolumn{3}{|l|}{ S. roseosporus } \\
\hline UA343 & A21978C producer & NRRL 15998 \\
\hline UA378 & $\Delta d p t D::$ ermE $r p s L 7$ & Miao et al. (2006b) \\
\hline SD301 & UA378 ::pCB301 & This study \\
\hline SD304 & UA378:: pCB304 & This study \\
\hline SD305 & UA378 : : pCB305 & This study \\
\hline SD306 & UA378:: pCB306 & This study \\
\hline SD307 & UA378:: pCB307 & This study \\
\hline SD308 & UA378::pCB308 & This study \\
\hline SD309 & UA378:: pCB309 & This study \\
\hline SD409 & UA378:: pCB409 & This study \\
\hline SD410 & UA378 : : pCB410 & This study \\
\hline \multicolumn{3}{|l|}{ E. coli } \\
\hline DH10B & $\begin{array}{l}\mathrm{F}^{-} \text {mcrA } \Delta(\text { mrr-hsdRMS-mcrBC }) \phi 80 \text { lacZ } \Delta \mathrm{M} 15 \Delta \text { lacX74 recA1 endA1 } \\
\text { araD139 } \Delta(\text { ara, leu }) 7697 \text { galU galK } \lambda^{-} \operatorname{rpsL}\left(\mathrm{Sm}^{\mathrm{R}}\right) \text { nupG }\end{array}$ & Invitrogen \\
\hline ML22 & DH10B : :pUZ8002 & Coëffet-Le Gal et al. (2006) \\
\hline \multicolumn{3}{|l|}{ Plasmids } \\
\hline BW25113 & lacI $^{\mathrm{q}} \mathrm{rrnB}_{\mathrm{T} 14} \Delta l a c Z_{\mathrm{WJ} 16} h s d R 514 \Delta a r a B A D_{\mathrm{AH} 33} \Delta r h a B A D_{\mathrm{LD} 78}$ & Datsenko \& Wanner (2000) \\
\hline pRHB538 & $\operatorname{rpsL} r e p^{\mathrm{ts}} \mathrm{Am}^{\mathrm{r}}$ & Hosted \& Baltz (1997) \\
\hline pHM11a & $\mathrm{Hm}^{\mathrm{r}}$ oriT ermEp att $/$ int $^{\mathrm{IS} 117}$ & Motamedi et al. (1995) \\
\hline pRB04 & pHM11a::dptD & Miao et al. (2006b) \\
\hline pStreptoBAC V & BAC vector $\dagger$ oriT att/int ${ }^{\phi \mathrm{C} 31} \mathrm{Am}^{\mathrm{r}}$ & Miao et al. (2005) \\
\hline pCB307 & pCB301 $(\Delta d p t D 2$ A site $):: d p t D 2(\mathrm{CATTe})$ & This study \\
\hline pCB308 & pCB303 ( $\Delta d p t D 2$ C site $):: d p t D 2(\mathrm{CATTe})$ & This study \\
\hline pCB309 & pCB301 ( $\Delta$ dptD2 C site) $:: d p t D 2(\mathrm{CATTe}) \Delta 81172-81184$ & This study \\
\hline pCB310 & pCB303 $(\Delta d p t D 2$ A site $):: d p t D 2($ CATTe $)+81172-81184$ & This study \\
\hline pCB401 & pCB302::dptD2(CAT) $:: d p t D 2(\mathrm{Te})$ & This study \\
\hline pCB407 & pCB302::lptC4(CAT) $:: \operatorname{dptD2}(\mathrm{Te})$ & This study \\
\hline pCB409 & pCB302::dptD2(CA) ::dptD2 (TTe) & This study \\
\hline pCB410 & pCB302::lptC4(CA) ::dptD2(TTe) & This study \\
\hline
\end{tabular}

$\dagger \mathrm{BAC}$, bacterial artificial chromosome.

Miao et al., 2006b), suggested a relatively simple test system to explore the requirements for functional module and domain exchanges. The di-modular $d p t D, l p t D$ and $c d a P S 3$ genes can be split into individual modules, and ligated in different combinations to produce hybrid di-modules; these can be tested for efficiency of trans-complementation of a $S$. roseosporus $\Delta d p t D$ mutant. In this way, the interpeptide docking sequences can be kept constant by using the $3 \mathrm{mGlu}_{12}$ module from $d p t D$, and fusing it in different ways to the $\mathrm{Kyn}_{13}, \mathrm{Ile}_{13}$ and $\operatorname{Trp}_{11}$ modules from the $d p t D, \quad l p t D$ and $c d a P S 3$ genes, respectively. The efficiencies of lipopeptide production can be compared to the results of whole-subunit exchanges carried out previously. In describing the current experiments, we represent inter-domain linker regions as hyphens (e.g. CA-T-C-A-T-Te for DptD and related di-modules), and use : : to represent fusion sites (e.g. C-A-T::C-A-T-Te or CAT:: CATTe for fusion at the T-C linker). 
(a)

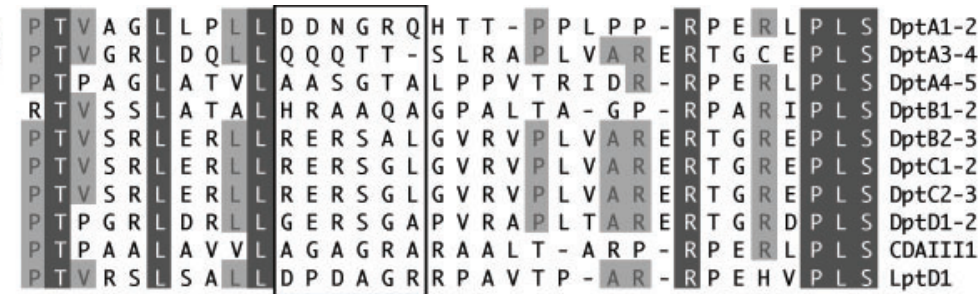

Module 12

Module 13

\section{T domain $\quad \mathrm{T}-\mathrm{C}$ linker $\quad \mathrm{C}$ domain}

(b)

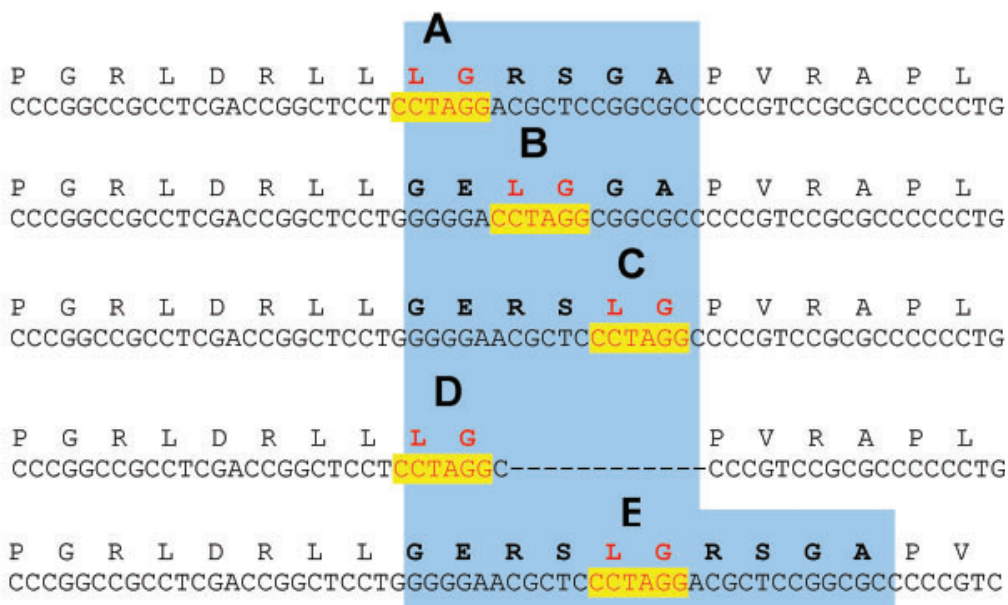

Fig. 2. $T-C$ linker sequences in daptomycin NRPSs, CdaPS3 and LptD, and structures of engineered T-C linkers. (a) Alignment of the TC linker regions of lipopeptide NRPSs. The boxed area depicts the proposed T-C linker region used for module fusions. (b) Introduction of an Avrll site in the A, B, C, D and $\mathrm{E}$ sites of the proposed $\mathrm{T}-\mathrm{C}$ linker region in DptD in strains SD307, SD304, SD308, SD309 and SD310, respectively. The shaded area depicts the linker region. Contraction and expansion of the T-C linkers $D$ and $E$ in strains SD309 and 310 are also shown.

The first experiments explored the simple fusion of the two $d p t D$ module coding sequences by inserting restriction endonuclease cleavage sites into the $\mathrm{T}-\mathrm{C}$ linker. From sequence comparisons between several T-C linker regions in the daptomycin NRPS proteins, LptD and CdaPS3, we noted a stretch of about 21 aa with little sequence conservation (Fig. 2a). The first six amino acids showed minimal conservation, so we predicted that they were at least part of the T-C linker. An AvrII restriction site was introduced in various positions of this proposed T-C linker coding sequence to generate three different new sequences (A, B and C; Fig. 2b), resulting in three sequential 2 aa substitutions in the protein. The recombinant plasmids were introduced into $S$. roseosporus UA378 $(\Delta d p t D)$ by conjugation and integrated at the IS117 attB site, and all recombinants produced daptomycin at similar high levels (Table 2). The results indicate that the 6 aa peptide is indeed part of a functional linker, and that the precise amino acid sequence is not critical for DptD function during daptomycin biosynthesis.

To further explore the flexibility of the linker region, we engineered linkers that were contracted or expanded by 4 aa (linkers D and E; Fig. 2b). Recombinants expressing DptD with these modified linkers produced the same yields of lipopeptides as those containing 2 aa substitutions (Table 2 ), and the same relative yields of individual lipopeptides (not shown). The results suggest that the T-C linker region in DptD has no obvious enzymic and structural function, other than connecting $\mathrm{T}$ and $\mathrm{C}$ domains.

BLAST analysis (http://www.ncbi.nlm.nih.gov/) of DptD against the TycC linker from Bacillus brevis ATCC 8185 indicated that the 6 aa segment of the T-C linker in DptD lies within the TycC linker, starting at position 4 . The TycC linker ends in the conserved proline also observed 3 aa from the right end in the sequences shown in Fig. 2(a) (Samel et al., 2007). Thus the DptD and other streptomy-

Table 2. Production yields of recombinant strains containing module fusions

\begin{tabular}{|llcc|}
\hline Strain & Module 13 & Linker $^{*}$ & $\begin{array}{c}\text { Lipopeptide yield } \\
\left(\mathbf{m g ~ l}^{\mathbf{1}}\right)\end{array}$ \\
\hline SD307 & Kyn & A & $111 \pm 12$ \\
SD304 & Kyn & B & $105 \pm 10$ \\
SD308 & Kyn & C & $111 \pm 8$ \\
SD309 & Kyn & D & $108 \pm 8$ \\
SD310 & Kyn & E & $129 \pm 10$ \\
SD306 & Trp & B & $134 \pm 9$ \\
SD305 & Ile/Val & B & $86 \pm 9$ \\
\hline
\end{tabular}

${ }^{\star}$ See Fig. 2 for linker structures. 
cete lipopeptide linkers in Fig. 2(a) may span 20-22 aa, providing additional sites for module fusions.

\section{Heterologous module fusions in DptD}

Module fusions were generated to link the DptD module for $3 \mathrm{mGlu}_{12}$ to the terminal modules for Trp ${ }_{11}$ and $\mathrm{Ile}_{13}$ from CDA and A54145 NRPSs, respectively (Fig. 1). The substrate specificities of these modules redirect the synthesis of daptomycin to incorporate $\operatorname{Trp}$ and Ile/Val, respectively, in place of $\mathrm{Kyn}_{13}$. Both modules were introduced with their $\mathrm{C}$-terminal $\mathrm{Te}$ domains in the CAT:: CATTe fusions using the splice site B in the T-C linker (Fig. 2b and Fig. 3). These hybrid subunits restored lipopeptide biosynthesis in strain UA378 deleted for $d p t D$ at yields similar to those produced by recombinants reconstructed to produce daptomycin (Table 2). LC-MS analysis of fermentation broths detected the mass ions indicative of hybrid compounds with $\operatorname{Trp}_{13}(1630,1644$ and 1658) and $\operatorname{Ile}_{13}(1543,1557,1571$ and 1585), respectively (Table 3 ). The mass ions $(1529,1543,1557$ and 1571) indicative of compounds containing $\mathrm{Val}_{13}$ were also detected in lower abundance, consistent with the fact that LptD can also incorporate Val at lower efficiency than Ile (Baltz et al., 2005; Miao et al., 2006b). Similar results were obtained when the splice site $\mathrm{C}$ was used to generate hybrid DptD subunits (not shown).

\section{Domain exchanges in DptD and the role of native TTe linkage}

Although exchanges of the complete CATTe module of the daptomycin NRPS with similar CATTe modules from the
CDA and A54145 pathways were successful, the availability of modules containing Te domains is limited. It would be useful to be able to generate hybrid CATTe modules by excising CA or CAT from CAT or CATE modules, and ligating at A-T or T-Te linkers (Fig. 3). To test this, we chose the CATE module for D-Asn 11 from $l p t C$ (Miao et al., 2006a; Fig. 1) to generate C-A-T::Te and C-A::T-Te fusions (Table 3, Fig. 3). As controls, similar homologous fusions were made to reconstruct the $\mathrm{Kyn}_{13}$ module (strains SD401 and SD409; Table 1). These plasmids containing the hybrid $d p t D$ genes were introduced into $S$. roseosporus UA378 and fermentations of recombinant strains were carried out. The strains expressing the reconstituted $\mathrm{Kyn}_{13} \mathrm{CAT}:: \mathrm{Te}$ and CA::TTe fusions produced the predicted lipopeptides. The recombinant containing the $\mathrm{Asn}_{13}$ CAT::Te fusion produced no detectable lipopeptides, but the strain with the CA::TTe fusion produced lipopeptides with ESI mass ions of 1558, 1572 and 1586 and UV maximal absorptions at 220 and $281 \mathrm{~nm}$, as expected for the three novel compounds containing $\mathrm{A}_{2} 1978 \mathrm{C}_{1-3}\left(\mathrm{Asn}_{13}\right)$, at yields of about 36$60 \mathrm{mg} \mathrm{l}^{-1}$. As a representative example, the structure of A21978C $\mathrm{C}_{1}\left(\mathrm{Asn}_{13}\right)$ was supported by its HR-MS data at $\mathrm{m} / \mathrm{z}$ 1558.7042 $[\mathrm{M}+\mathrm{H}]^{+}$. Furthermore, analysis of LC-MS/MS data revealed that the Asn residue was located in the depsipeptide ring, as expected (Fig. 4).

\section{Biological activities of hybrid peptides}

While most of the daptomycin derivatives were very active against Staph. aureus 42, they were slightly less potent than daptomycin. The MIC of daptomycin against Staph. aureus 42 is $0.5-1 \mu \mathrm{g} \mathrm{ml}^{-1}$, whereas the MICs of the daptomycin

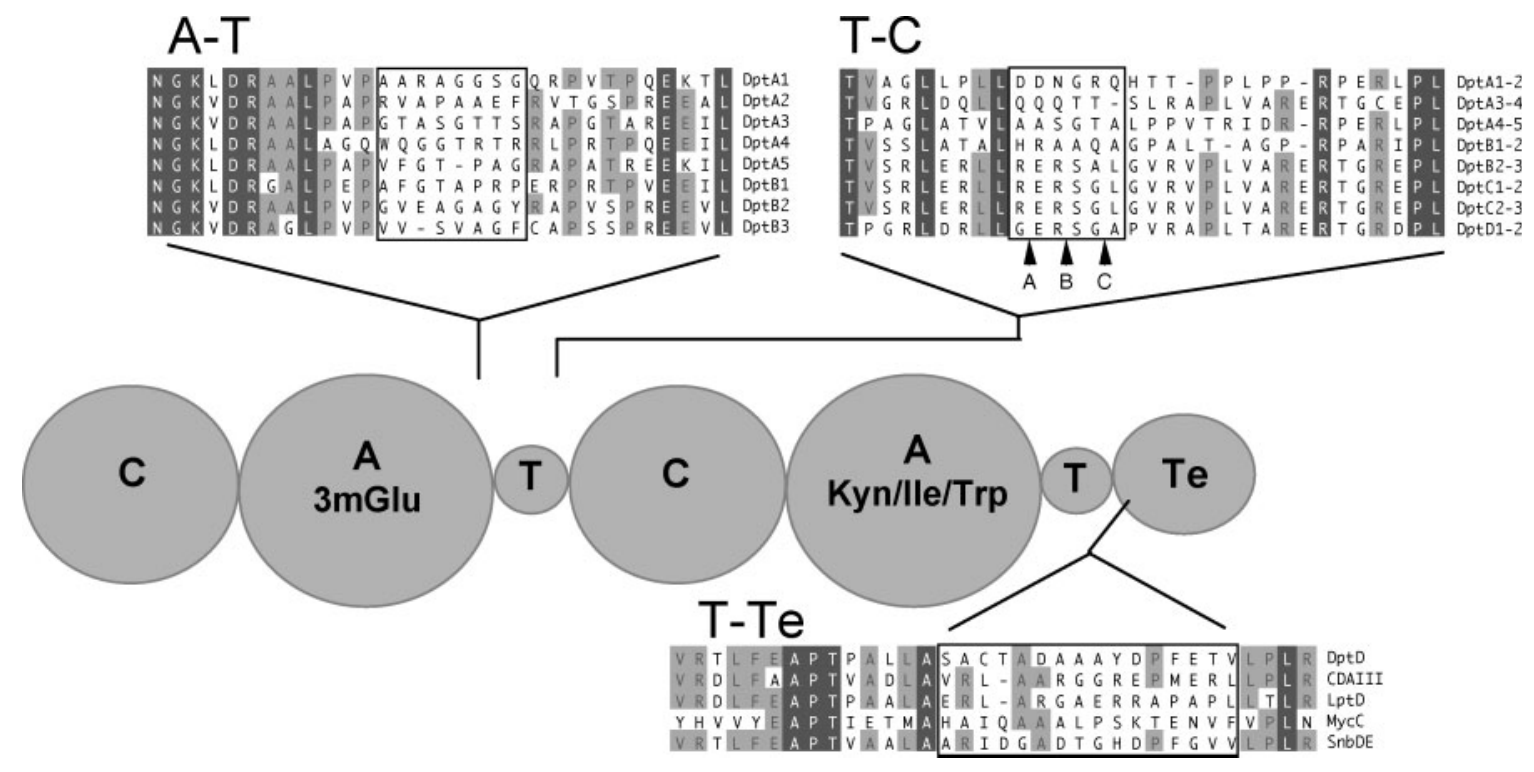

Fig. 3. Inter-domain linkers in NRPSs. Domain organization of DptD, LptD and CdaPS3 (CAT-CATTe). Alignments of the A-T linker, the T-C linker and the T-Te linker regions. Proposed linkers are boxed. 
Table 3. Production of lipopeptides by recombinants containing reconstructed or hybrid $d p t D$ genes

The module and domain fusions of daptomycin NRPS using domains from $d p t D$ (Kyn), lptD (Ile), cdaPS3 (Trp) and lptC (Asn) were constructed as described in Methods and in Table 1. The fusion sites are marked by ::. Fermentation broths of recombinants carrying plasmids encoding hybrid subunits were analysed by LC-MS. The heterologous domains from the A54145 and CDA pathways are italicized.

\begin{tabular}{|c|c|c|c|}
\hline Strain & Fusion scheme & Predicted mass ions & Mass ions detected \\
\hline SD304 & $\mathrm{CAT}:: \mathrm{CA}_{\mathrm{Kyn}} \mathrm{Te}$ & $1634,1648,1662$ & + \\
\hline SD305 & $\mathrm{CAT}:: C A_{I l e} T e$ & $1543,1557,1571,1585$ & + \\
\hline SD306 & CAT : : $C A_{T r p} T e$ & $1630,1644,1658$ & + \\
\hline SD401 & CAT : : $\mathrm{CA}_{\mathrm{Kyn}} \mathrm{T}:: \mathrm{Te}$ & $1634,1648,1662$ & + \\
\hline SD407 & CAT $:: C A_{A s n} T:: \mathrm{Te}$ & $1558,1572,1586$ & - \\
\hline SD409 & CAT $:: \mathrm{CA}_{\mathrm{Kyn}}:: \mathrm{TTe}$ & $1634,1648,1662$ & + \\
\hline SD410 & CAT $:: C A_{A s n}:: \mathrm{TTe}$ & $1558,1572,1586$ & + \\
\hline
\end{tabular}

derivatives were $1 \mu \mathrm{g} \mathrm{ml}^{-1}$ for the $\operatorname{Trp}_{13}$ derivative and 2$4 \mu \mathrm{g} \mathrm{ml}^{-1}$ for the $\mathrm{Ile}_{13}$ derivative. This is consistent with results obtained previously with heterologous wholesubunit exchanges (Nguyen et al., 2006a; Miao et al., 2006b). All three Asn $_{13}$ derivatives had MICs of $128 \mu \mathrm{g}$ $\mathrm{ml}^{-1}$, further emphasizing the critical importance of position 13 for antibacterial activity (Nguyen et al., 2006b; Miao et al., 2006b).

(a)

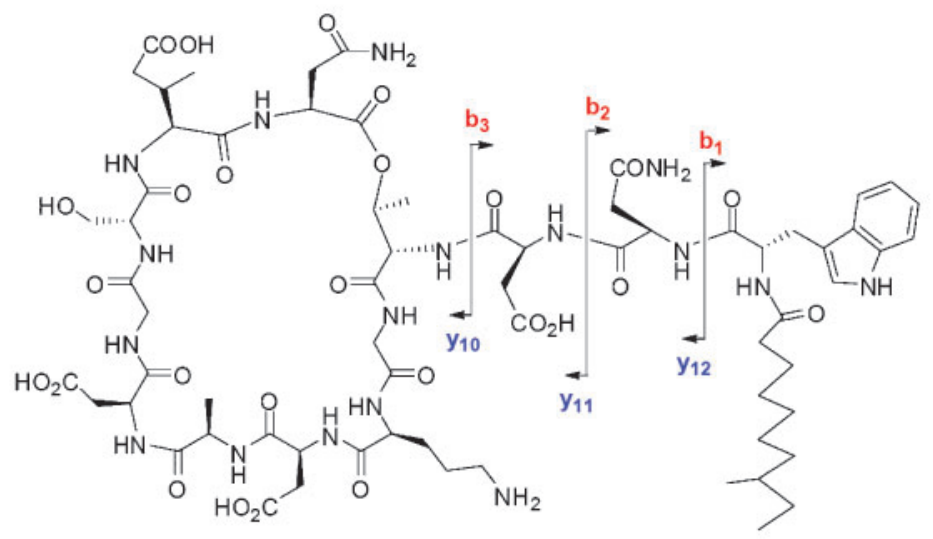

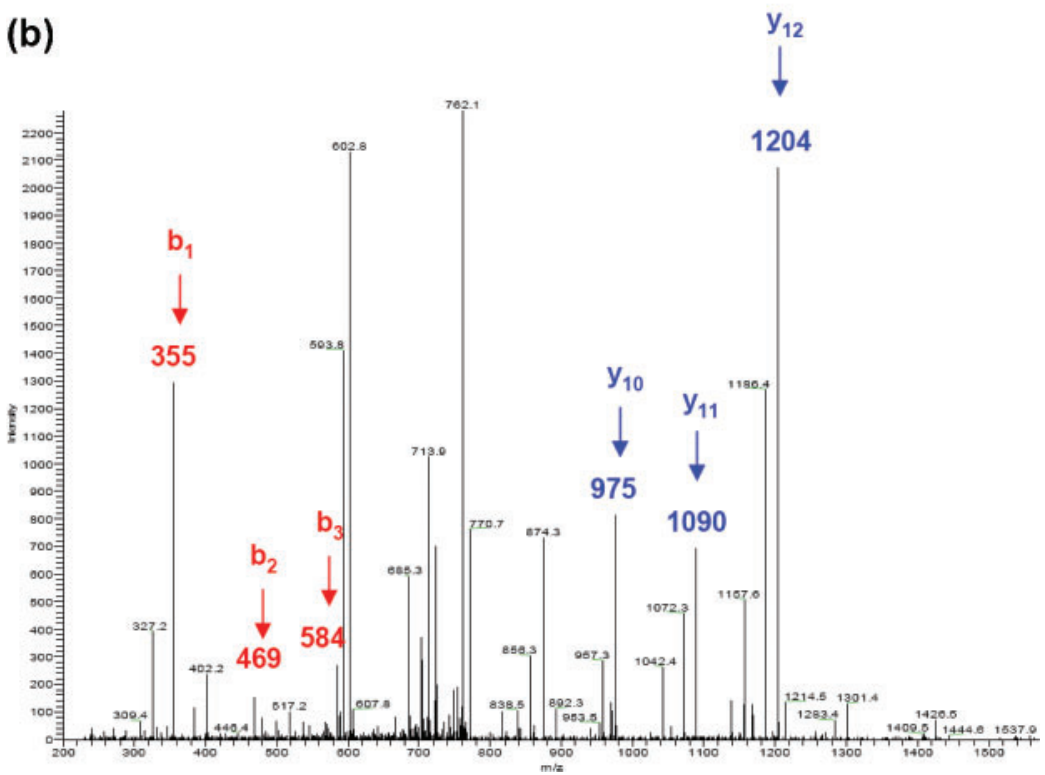

Fig. 4. $L C-M S / M S$ analysis of $A 21978 C_{1}$ $\left(\mathrm{Asn}_{13}\right)$ produced by strain SD410. (a) Chemical structure of $\mathrm{A}_{21978 \mathrm{C}_{1}}\left(\mathrm{Asn}_{13}\right)$ and six characteristic product ions $b_{1}-b_{3}$ and $\mathrm{y}_{10} \mathrm{y}_{12}$. (b) MS/MS spectrum of $\mathrm{A} 21978 \mathrm{C}_{1-}$ $\left(\mathrm{Asn}_{13}\right)$ and assignment of key product ions. 


\section{DISCUSSION}

It is now feasible to reprogramme the amino acid specificity of NRPS multi-enzymes, but the factors determining high-efficiency in vivo productivity from hybrid enzymes are poorly understood. This needs to be addressed to link the application of combinatorial biosynthesis to industrial-scale fermentation production. In the present work, we addressed the efficiency of producing hybrid lipopeptides related to daptomycin by fusing complete modules at $\mathrm{T}-\mathrm{C}$ linkers in the $\mathrm{DptD}$ subunit. We found that neither the sequence nor the length (within $\pm 4 \mathrm{aa}$ ) of a segment of the inter-module T-C linker is important for catalytic efficiency of DptD. This linker may simply act as a tether which ensures that domains are aligned and in close proximity.

The flexible T-C linker nucleotide sequences containing inserted restriction endonuclease sites were exploited to construct hybrid genes to redirect lipopeptide biosynthesis to produce hybrid compounds containing $\operatorname{Trp}_{13}$ or $\mathrm{Ile}_{13} /$ $\mathrm{Val}_{13}$ at levels comparable to those produced by recombinants producing the native daptomycin factors. This compares favourably with yields obtained using wholesubunit exchanges to produce the same hybrid molecules at about $50 \%$ and $25 \%$ of controls, respectively (Miao et al., 2006b; Coëffet-Le Gal et al., 2006). The high productivity of recombinant $S$. roseosporus strains containing heterologous module exchanges may be due in part to the use of homologous inter-peptide docking sites and promiscuous Te domains, but also must be due in part to the inherent flexibility of the inter-module linker. Thus two advantages of using module exchange, as opposed to subunit exchange, are that it conserves the natural inter-peptide docking sites required for efficient communication between $\mathrm{DptBC}$ and $\mathrm{DptD}$, and it provides a mechanism to expand the number of substitutions at position 13, which is important for antibacterial activity (Nguyen et al., 2006a; Miao et al., 2006b).

To further explore the engineering of the daptomycin biosynthetic pathway at the terminal module, we made fusions at $\mathrm{T}$-C linkers, keeping the $3 \mathrm{mGlu}_{12}$ module intact, coupled with fusions at A-T or T-Te linkers to exchange the CA or CAT at position 13. Of these, the CAT::CA::TTe double fusion, using the C-A di-domain from the D-Asn 11 (CATTe) module of A54145 was successful, whereas the CAT::CAT::Te double fusion did not yield product. This is consistent with previous work (Zhou et al., 2006) that demonstrated a functional interaction between $\mathrm{T}$ and $\mathrm{Te}$ in $\mathrm{T}$-Te di-domains. $\mathrm{T}$ domains from typical CAT modules might not be expected to carry out this function efficiently, if at all. Thus maintaining the integrity of the T-Te di-domain may be an important consideration in engineering terminal modules containing Te domains.

The fermentation yield of the recombinant containing the CAT : : CA : : TTe double fusion in $d p t D$ to insert $A_{13} n_{13}$ was about $30-50 \%$ of the yields obtained with strains contain- ing single module fusions. This promising approach provides a route to insert other amino acids at the important position 13. Suboptimal fermentation yields of recombinants may be addressed by focused mutagenesis, as has been demonstrated in the engineering of the mixed polyketide-peptide andrimid (Fischbach et al., 2007).

\section{ACKNOWLEDGEMENTS}

We thank N. Cotroneo for MIC determinations. This work was supported by Cubist Pharmaceuticals Inc. and all authors were employed by Cubist during the execution of this work.

\section{REFERENCES}

Arbeit, R. D., Maki, D., Tally, F. P., Campanaro, E. \& Eisenstein, B. I. (2004). The safety and efficacy of daptomycin for the treatment of complicated skin and skin-structure infections. Clin Infect Dis 38, 1673-1681.

Baltz, R. H. (2008). Biosynthesis and genetic engineering of lipopeptide antibiotics related to daptomycin. Curr Top Med Chem 8, 618-638.

Baltz, R. H., Miao, V. \& Wrigley, S. W. (2005). Natural products to drugs: daptomycin and related lipopeptide antibiotics. Nat Prod Rep 22, 717-741.

Challis, G. L., Ravel, J. \& Townsend, C. A. (2000). Predictive, structure-based model of amino acid recognition by nonribosomal peptide synthetase adenylation domains. Chem Biol 7, 211-224.

Coëffet-Le Gal, M.-F., Thurson, L., Rich, P., Miao, V. \& Baltz, R. H. (2006). Complementation of daptomycin $d p t A$ and $d p t D$ deletion mutations in-trans and production of hybrid lipopeptide antibiotics. Microbiology 152, 2993-3001.

Datsenko, K. A. \& Wanner, B. (2000). One-step inactivation of chromosomal genes in Escherichia coli K-12 using PCR products. Proc Natl Acad Sci U S A 97, 6640-6645.

Debono, M., Barnhart, M., Carrell, C. B., Hoffmann, J. A., Occolowitz, J. L., Abbott, B. J., Fukuda, D. S., Hamill, R. L., Biemann, K. \& Herlihy, W. C. (1987). A21978C, a complex of new acidic peptide antibiotics: isolation, chemistry, and mass spectral structure elucidation. $J$ Antibiot 40, 761-777.

Doekel, S. \& Marahiel, M. A. (2000). Dipeptide formation on engineered hybrid peptide synthetases. Chem Biol 7, 373-384.

Eppelmann, K., Stachelhaus, T. \& Marahiel, M. A. (2002). Exploitation of the selectivity-conferring code of nonribosomal peptide synthetases for the rational design of novel peptide antibiotics. Biochemistry 41, 9718-9726.

Fischbach, M. A. \& Walsh, C. T. (2006). Assembly-line enzymology for polyketide and nonribosomal peptide antibiotics: logic, machinery, and mechanisms. Chem Rev 106, 3468-3496.

Fischbach, M. A., Lai, J. R., Roche, E. D., Walsh, C. T. \& Liu, D. R. (2007). Directed evolution can rapidly improve the activity of chimeric assembly-line enzymes. Proc Natl Acad Sci U S A 104, 11951-11956.

Fowler, V. G., Boucher, H. W., Corey, G. R., Abrutyn, E., Karchmer, A. W., Rupp, M. E., Levine, D. P., Chambers, H. F., Tally, F. P. \& other authors (2006). Daptomycin versus standard therapy for bacteremia and endocarditis caused by Staphylococcus aureus. N Engl J Med 355, 653-655.

Grünewald, J., Sieber, S. A., Mahlert, C., Linne, U. \& Marahiel, M. A. (2004). Synthesis and derivation of daptomycin: a chemoenzymatic 
route to acidic lipopeptide antibiotics. J Am Chem Soc 126, 1702517031.

Hojati, Z., Milne, C., Harvey, B., Gordon, L., Borg, M., Flett, F., Wilkinson, B., Sidebottom, P. J., Rudd, B. A. M. \& other authors (2002). Structure, biosynthetic origin, and engineered biosynthesis of calcium-dependent antibiotics from Streptomyces coelicolor. Chem Biol 9, 1175-1187.

Hosted, T. J. \& Baltz, R. H. (1997). Use of $r p s L$ for dominance selection and gene replacement in Streptomyces roseosporus. J Bacteriol 179, 180-186.

Huber, F. M., Pieper, R. L. \& Tietz, A. J. (1988). The formation of daptomycin by supplying decanoic acid to Streptomyces roseosporus cultures producing the antibiotic complex A21978C. J Biotechnol 7, 283-292.

Kohli, R. M. \& Walsh, C. T. (2003). Enzymology of acyl chain macrocyclization in natural product biosynthesis. Chem Commun 297-307.

Kopp, F., Grünewald, J., Mahlert, C. \& Marahiel, M. A. (2006). Chemoenzymatic design of acidic lipopeptide hybrids: new insights into the structure-activity relationship of daptomycin and A54145. Biochemistry 45, 10474-10481.

Miao, V., Coëffet-Le Gal, M.-F., Brian, P., Brost, R., Penn, J., Whiting, A., Martin, S., Ford, R., Parr, R. \& other authors (2005). Daptomycin biosynthesis in Streptomyces roseosporus: cloning and analysis of the gene cluster and revision of peptide stereochemistry. Microbiology 151, 1507-1523.

Miao, V., Brost, R., Chapple, J., She, K., Coëffet-Le Gal, M.-F. \& Baltz, R. H. (2006a). The lipopeptide antibiotic A54145 biosynthetic gene cluster from Streptomyces roseosporus. J Ind Microbiol Biotechnol 33, 129-140.

Miao, V., Coëffet-Le Gal, M.-F., Nguyen, K., Brian, P., Penn, J., Whiting, A., Steele, J., Kau, D., Martin, S. \& other authors (2006b). Genetic engineering in Streptomyces roseoporus to produce hybrid lipopeptide antibiotics. Chem Biol 13, 269-276.

Mootz, H. D., Schwarzer, D. \& Marahiel, M. A. (2000). Construction of hybrid peptide synthetases by module and domain fusions. Proc Natl Acad Sci U S A 97, 5848-5853.

Mootz, H. D., Kessler, N., Linne, U., Eppelmann, K., Schwarzer, D. \& Marahiel, M. A. (2002). Decreasing the ring size of a cyclic nonribosomal peptide antibiotic by in-frame module deletion in the biosynthetic genes. J Am Chem Soc 124, 10980-10981.

Motamedi, H., Shafiee, A. \& Cai, S. J. (1995). Integrative vectors for heterologous gene expression in Streptomyces spp. Gene 160, 25-31.

Nguyen, K. T., Kau, D., Gu, J.-Q., Brian, P., Wrigley, S. K., Baltz, R. H. \& Miao, V. (2006a). A glutamic acid 3-methyltransferase encoded by an accessory gene locus important for daptomycin biosynthesis in Streptomyces roseosporus. Mol Microbiol 61, 1294-1307.

Nguyen, K. T., Ritz, D., Gu, J.-Q., Alexander, D., Chu, M., Miao, V., Brian, P. \& Baltz, R. H. (2006b). Combinatorial biosynthesis of lipopeptide antibiotics related to daptomycin. Proc Natl Acad Sci U S A 103, 17462-17467.

Samel, S. A., Schoenafinger, G., Knappe, T. A., Marahiel, M. A. \& Essen, L.-O. (2007). Structural and functional insights into a peptide bond-forming bidomain from a nonribosomal peptide synthetase. Structure 15, 781-792.

Shen, B., Du, L., Sanchez, C., Edwards, D. J., Chen, M. \& Murrell, J. M. (2002). Cloning and characterization of the bleomycin biosynthetic gene cluster from Streptomyces verticillus ATCC15003. J Nat Prod 65, 422-431.

Sieber, S. A. \& Marahiel, M. A. (2005). Molecular mechanisms underlying nonribosomal peptide synthesis: approaches to new antibiotics. Chem Rev 105, 715-738.

Stachelhaus, T. \& Walsh, C. T. (2000). Mutational analysis of the epimerization domain in the initiation module PheATE of gramicidin S synthetase. Biochemistry 39, 5775-5787.

Stachelhaus, T., Schneider, A. \& Marahiel, M. A. (1995). Rational design of peptide antibiotics by targeted replacement of bacterial and fungal domains. Science 269, 69-72.

Stachelhaus, T., Hüser, A. \& Marahiel, M. A. (1996). Biochemical characterization of the peptidyl carrier protein (PCP), the thiolation domain of multifunctional peptide synthetases. Chem Biol 3, 913-921.

Stachelhaus, T., Mootz, H. D., Bergendahl, V. \& Marahiel, M. A. (1998). Peptide bond formation in nonribosomal peptide biosynthesis. Catalytic role of the condensation domain. J Biol Chem 273, 22773-22781.

Stachelhaus, T., Mootz, H. D. \& Marahiel, M. A. (1999). The specificity-conferring code of adenylation domains in nonribosomal peptide synthetases. Chem Biol 6, 493-505.

Yakimov, M. M., Giuliano, L., Timmis, K. N. \& Golyshin, P. N. (2000). Recombinant acylheptapeptide lichenysin: high level of production by Bacillus subtilis cells. J Mol Microbiol Biotechnol 2, 217-224.

Zhang, Y., Buchholz, F., Muyrers, J. P. P. \& Stewart, F. (1998). A new logic for DNA engineering using recombination in Escherichia coli. Nat Genet 20, 123-128.

Zhou, Z., Lai, J. R. \& Walsh, C. T. (2006). Interdomain communication between the thiolation and thioesterase domains of EntF explored by combinatorial mutagenesis and selection. Chem Biol 13, 869-879.

Edited by: L. Heide 